\title{
Transcriptional regulation of human sperm-associated antigen 16 gene by S-SOX5
}

\author{
Ling Zhang ${ }^{1,2^{*}+}$, Yunhao Liu ${ }^{1 \dagger}$, Wei Li ${ }^{2}$, Qiaoling Zhang ${ }^{3}$, Yanwei Li ${ }^{2,4}$, Junpin Liü ${ }^{2,5}$, Jie Min ${ }^{1}$, Chaofan Shuang ${ }^{1}$, \\ Shizheng Song ${ }^{1}$ and Zhibing Zhang ${ }^{2^{*}}$
}

\begin{abstract}
Background: The mammalian sperm-associated antigen 16 gene (Spag16) uses alternative promoters to produce two major transcript isoforms (Spag16L and Spag16S) and encode proteins that are involved in the cilia/flagella formation and motility. In silico analysis of both mouse and human SPAG16L promoters reveals the existence of multiple putative SOX5 binding sites. Given that the SOX5 gene encodes a 48-kDa transcription factor (S-SOX5) and the presence of putative SOX5 binding sites at the SPAG16L promoter, regulation of SPAG16L expression by S-SOX5 was studied in the present work.
\end{abstract}

Results: S-SOX5 activated human SPAG16L promoter activity in the human bronchial epithelia cell line BEAS-2B cells. Mutation of S-SOX5 binding sites abolished the stimulatory effect. Overexpression of S-SOX5 resulted in a significant increase in the abundance of SPAG16L transcripts whereas silencing of S-SOX5 by RNAi largely reduced the SPAG16L expression. Chromatin immunoprecipitation assays showed that S-SOX5 directly interacts with the SPAG16L promoter.

Conclusion: S-SOX5 regulates transcription of human SPAG16L gene via directly binding to the promoter of SPAG16L. It has been reported that expression of sperm-associated antigen 6 (SPAG6), encoding another axonemal protein, is activated by S-SOX5. Therefore, S-SOX5 may regulate formation of motile cilia/flagella through globally mediating expression of genes encoding axonemal proteins.

Keywords: S-SOX5, SPAG16L, Transcriptional regulation, Central apparatus, Cilia

\section{Background}

The family of Sox transcription factors is defined by the presence of a conserved high mobility-group (HMG) domain that mediates DNA-binding and is highly similar to that of the sex-determining region (SRY) protein $[1,2]$. Based on phylogenic analysis of HMG domain sequences and full-length protein sequences/functional features, Sox genes are classified into 10 groups from A to J [3]. They display distinct tissue-specific expression patterns and have been implicated in regulation of a wide range of developmental processes [1]. SOX proteins exert gene activation or repression by binding to a consensus DNA

\footnotetext{
*Correspondence: zhangling@wust.edu.cn;

zhibing.zhang@vcuhealth.org

${ }^{\dagger}$ Ling Zhang and Yunhao Liu are contributed equally to this work

2 Department of Obstetrics and Gynecology, Virginia Commonwealth

University, Richmond, VA 23298, USA

Full list of author information is available at the end of the article
}

motif, with or without aid of other transcription factors $[1,4]$. Available evidence indicates that a particular SOX protein can mediate expression of various target genes through recognizing different binding sites during the formation of many tissues $[1,5]$. Selection of specific target genes by SOX proteins depends on flanking sequences of the consensus core, homo-/hetero-dimerization of SOX proteins at recognition sites and association with other transcription factors $[1,6]$.

The SOXD group is composed of SOX5, SOX6 and SOX13 [1]. Human SOX5 is primarily expressed in the short (S-SOX5) and long form (L-SOX5) of transcripts [7, 8]. $L$-SOX 5 CDNA is predicted to encode a 763 -aminoacid protein that exceeds S-SOX5 by 416 residues [8]. $\mathrm{S}$-SOX5, which lacks $\mathrm{N}$-terminal domain required for dimerization with other SOXD proteins, is predominantly detected in testis and brain while L-SOX5 is expressed in multiple tissues including testis, heart, liver 
and skeletal muscle $[7,8]$. The difference in the protein structure and tissue distribution between the two forms of SOX5 implies distinct biological functions for these isoforms. The two SOX5 isoforms are conserved in mouse [9]. Mouse S-Sox5 was originally cloned from testis [10]. The restricted presence of mouse orthologue S-SOX5 proteins in round spermatids and regulation of testis-related gene expression by S-SOX5 suggests that S-SOX5 plays a specialized role in spermatogenesis within the testis [10-13]. Later studies demonstrated that mouse S-SOX5 is also expressed in the lung and brain, tissues bearing motile cilia [9], and it is capable of activating expression of sperm-associated antigen 6 gene (Spag6), whose translated product is enriched in the tissues with motile cilia, particularly in the testis [9].

Mammalian sperm-associated antigen 16 (Spag16) is the orthologue of Chlamydomonas reinhardtii pf20 that encodes an axonemal protein essential for flagellar motility. Chlamydomonas mutants carrying pf 20 mutation display paralyzed flagella with defects in axonemal central apparatus [14]. Both mouse and human SPAG16 genes are expressed as two major transcripts of 1.4 and $2.5 \mathrm{~kb}$ with different expression patterns. The human $1.4 \mathrm{~kb}$ transcript was detected in multiple tissues whereas the human 2.5 transcript was highly expressed in testis $[15,16]$. Mouse $2.5 \mathrm{~kb}$ transcript has a similar tissue distribution as the human orthologue; however, the $1.4 \mathrm{~kb}$ transcript is only present in mouse testis [17]. The translated $71 \mathrm{kDa}$ (SPAG16L) and $35 \mathrm{kDa}$ (SPAG16S) proteins have different locations and functions in male germ cells. SPAG16L is located in the axoneme central apparatus of sperms and plays a crucial role in sperm motility. Besides the similar localization as SPAG16L, SPAG16S is also present in the nucleus of post-meiotic germ cells and seems to be essential for viability of these cells during spermatogenesis $[16,18,19]$.

Given that both S-SOX5 and SPAG16L are present in tissues containing cells with motile cilia/flagella, it is hypothesized that expression of $S P A G 16 L$ is regulated by S-SOX5. In the present work, we report bioinformatic and biochemical characterization of the human SPAG16L promoter. The in silico prediction showed multiple putative binding sites for SOX5 in the SPAG16L promote region. The empirical evidence revealed that S-SOX5 activates expression of $S P A G 16 L$ via direct interaction with SOX5 binding sites at the SPAG16L promoter.

\section{Results}

\section{S-SOX5 stimulates human SPAG16L promoter in BEAS-2B}

cells

We have previously used the ConSite program to predict transcription factors of human SPAG16L and found multiple putative SOX5-binding sites in the 2-kb proximal promoter region of SPAG16L [9]. To explore the influence of S-SOX 5 on expression of SPAG16L, a transcriptional luc fusion was made by cloning a 2-kb DNA fragment containing the $S P A G 16 L$ proximal promoter into pGL3-basic vector. The resultant construct (SPAG16/pGL3) was cotransfected with S-SOX5 expression plasmids (S-SOX5/ pcDNA3) into human bronchial epithelial cells BEAS-2B and the relative luciferase activity was measured. Endogenous expression of S-SOX5 has been reported in BEAS-2B cells, suggesting this cell line can be used to study S-SOX5mediated gene regulation [9]. The basal level of SPAG16L expression was detected, which is about $10 \times$ higher than that of the control empty pGL3-basic plasmids. When cotransfected with S-SOX5, the relative luciferase activity was elevated about 100 -folds (Fig. 1). These results indicate that S-SOX5 stimulates transcription of human SPAG16L.

\section{Levels of SPAG16L mRNA are elevated by exogenous S-SOX5}

The effect of S-SOX 5 on the expression level of SPAG16L mRNA in BEAS-2B cells was examined. To this end, BEAS-2B cells stably expressing S-SOX5 (S-SOX5/ pcDNA3) or infected with S-SOX5 adenovirus (Ad/SSOX5) were generated, and expression of S-SOX5 protein in these cells was confirmed by Western blot analysis. Even though, S-SOX5 was undetected in the controls, the S-SOX5 protein $(48 \mathrm{kDa})$ was produced in both cell lines and clearly increased when infected with S-SOX5 adenovirus (Fig. 2a). To measure the relative abundance of SPAG16L mRNA in the same cells, real-time PCR was performed. As expected, the relative level of SPAG16L mRNA in both cell types was significantly higher than that in the control (Fig. 2b). The results show that exogenous S-SOX5 is able to increase SPAG16L mRNA levels.

\section{Knockdown of S-SOX5 in BEAS-2B cells reduces expression of SPAG16L}

To further investigate the regulatory role of S-SOX5 on SPAG16L expression, two human SOX5 RNAi constructs that respectively target portion 225-246 and 1109-1130 of the SOX5 transcript were used [9]. The BEAS-2B cells were co-transfected with these constructs or SOX5 expression plasmids (S-SOX5/pcDNA3). The efficiency of the SOX5 RNAi plasmids was examined by Western blot analysis. Both RNAi constructs were able to decrease S-SOX5 protein levels, but the construct targeting portion 1109-1130 of the SOX5 transcript led to a remarkable reduction of S-SOX5 (Fig. 3a, b). Moreover, the abundance of SPAG16L mRNA in the BEAS-2B cells was measured by real-time PCR. It was decreased correspondingly with two different RNAi constructs (Fig. 3c). These data suggest that expression of SPAG16L depends on the activity of S-SOX5 protein. 

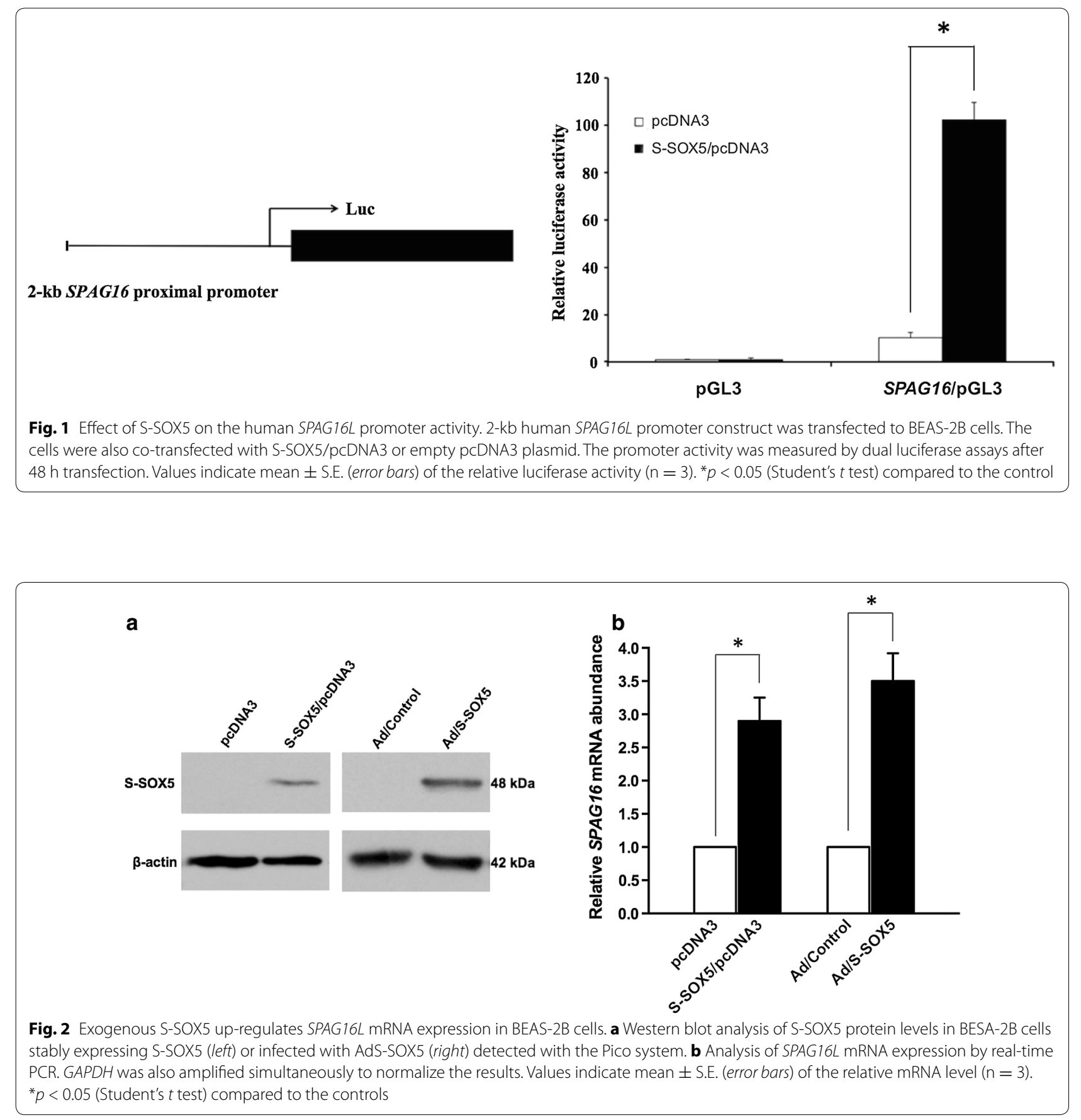

\section{S-SOX5 binds to the human SPAG16L promoter}

To study the molecular interaction between S-SOX5 and the SPAG16L promoter, BEAS-2B cells were infected with S-SOX5 adenovirus (AdS-SOX5) and ChIP assay was conducted with an antibody specifically against S-SOX5 or rabbit IgG. Two primer sets flanking putative SOX5 binding sites were designed; as a control, another primer set located about $3 \mathrm{~kb}$ upstream of the transcriptional start site without flanking any SOX5 binding site was also designed (Fig. 4A). Compared to using the rabbit IgG, more PCR products were amplified by two PCR primer sets flanking putative SOX5 binding sites when ChIP was performed using the SOX5 antibody (Fig. 4B, b, c). However, the control primer set was unable to amplify more specific DNA fragments (Fig. 4B, a). The result was consistent with the statistical analysis of the 

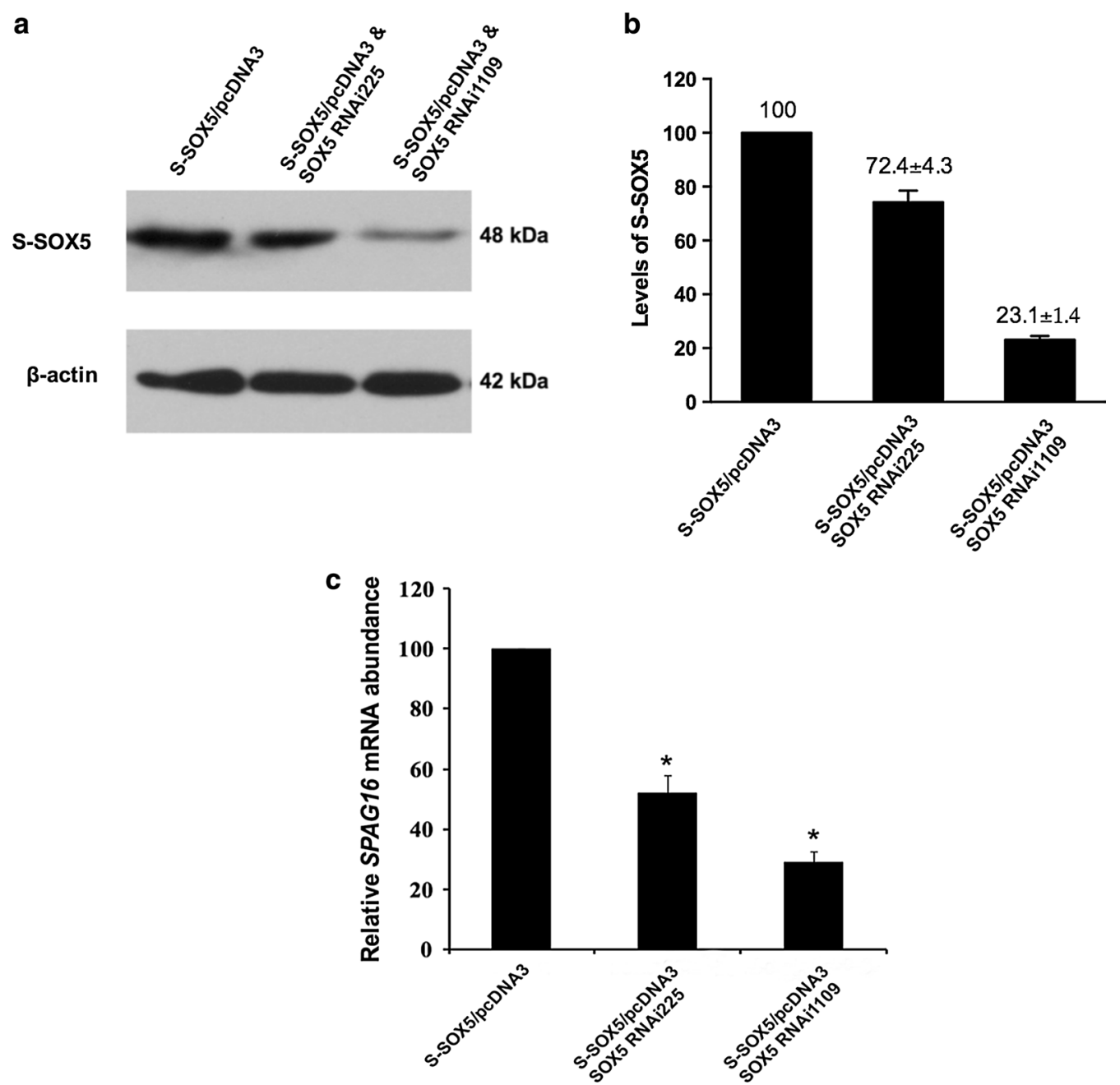

Fig. 3 Knockdown of S-SOX5 in BEAS-2B cells by RNAi results in decrease of SPAG16L mRNA. a Western blot analysis of S-SOX5 proteins in BEAS-2B cells stably expressing two RNAi constructs. b After RNAi treatment, the levels of S-SOX5 in BEAS-2B cells were estimated by analysing intensity of protein bands using ImageJ software. c Analysis of SPAG16L mRNA expression by real-time PCR in BEAS-2B cells transfected with two SOX5 RNAi constructs. Silencing of S-SOX5 by RNAi reduced expression of SPAG16L. Values indicate mean \pm S.E. (error bars) of the relative $m R N A$ level $(n=3)$. ${ }^{*} p<0.05$ (Student's $t$ test) compared to the control

relative abundance of the PCR products (Fig. 4C). These results suggest that $\mathrm{S}-\mathrm{SOX} 5$ is capable of binding to the promoter of SPAG16L.

\section{Mutation of SOX 5 binding sites abolishes activation of the SPAG 16L promoter in BEAS-2B cells}

To study if S-SOX5 activates SPAG16L transcription via interacting with the SOX5 binding sites, a 300-bp SPAG16L promoter construct encompassing two putative SOX5 binding sites was generated. The resultant transcriptional fusion construct was transfected into BEAS-2B cells and relative luciferase activity was analyzed. The SPAG16L promoter showed basal level activity which is about $10 \times$ higher than that of the control
(pGL3 only). As expected, co-transfection of S-SOX5 largely stimulated the $S P A G 16 L$ promoter activity when both putative SOX5 binding sites (P-I \& P-II) were present (Fig. 5). Mutation in the P-II site had little effect on the elevation of SPAG16L promoter activity, suggesting that the P-II site is not required for S-SOX5-mediated activation of SPAG16L. Interestingly, mutations in either the P-I site or both sites (P-I \& P-II) led to higher baseline promoter activities; however, the promoter activities were not increased in the presence of S-SOX5 (Fig. 5). The results suggest that the P-I site probably contributes to the repression of SPAG16L by unknown factor(s) in the absence of S-SOX5 and is also essential for activation of SPAG16L by S-SOX5. 


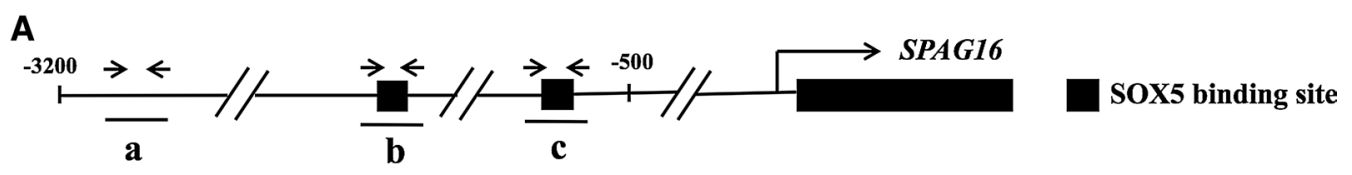

B

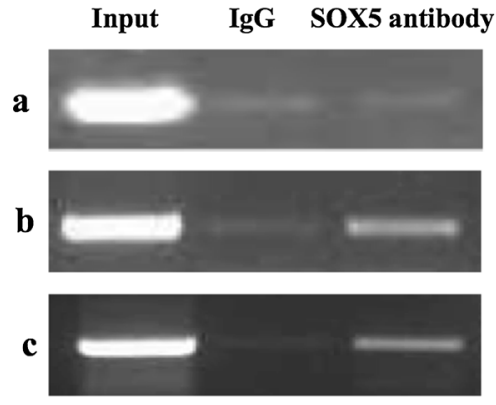

C

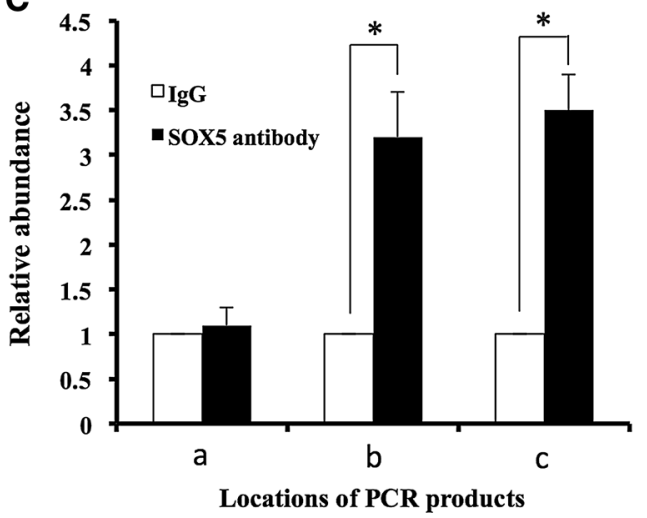

Fig. 4 S-SOX5 associates with the human SPAG16L promoter as revealed by ChIP assay. A Schematic representation of the human SPAG16L proxima promoter and the regions $(a-c)$ amplified by ChIP primers used in this study. Arrows show the location of the primers. B Representative ChIP assay results with BEAS-2B cells infected by AdS-SOX5 using a control rabbit lgG or an antibody specifically against SOX5. $a$ with a primer set not flanking any potential SOX5 binding sites; $b$ and $c$, with primer sets flanking potential SOX5 binding sites. C qPCR quantification of ChIP products. DNA recovered from ChIP was used as a template for real-time PCR analysis. Data shown are mean \pm S.E. (error bars) of three independent replicates. ${ }^{*} p<0.05$ (Student's $t$ test) compared to the normal rabbit IgG pulldown group

\section{Discussion}

Cilia are evolutionarily conserved, filamentous cellular structures that are present on the cell surface and have been implicated in the sensing of environmental signals and cellular motility [20]. Based on the axonemal architectures, cilia can be classified into two major forms: " $9+0$ " and " $9+2$ " axonemal arrangements [20]. Primary cilia contain a " $9+0$ " axoneme and usually non-motile, and detect mechanical and chemical signals from the surrounding environment. Dysfunction of primary cilia can lead to various human diseases including primary ciliary dyskinesia, polycystic kidney disease and retinal degeneration [21]. Motile cilia have a " $9+2$ " axoneme that is composed of nine doublet microtubules and a central pair of microtubules. The associated structures of the " $9+2$ " axoneme such as radial spokes and dynein arms are crucial for mediation of cilia motility [20]. Motile cilia are widely present in mammalian tissues including trachea, brain, spinal canal and sperm. Defects in motile cilia have been linked to diverse symptoms including hydrocephalus, sinusitis and bronchiectasis, situs inversus and male infertility [22].

Fine-tuned regulatory mechanisms mediate expression of stage-specific genes for the formation of distinct types of cilia during ciliogenesis. The sophisticated genetic program of ciliogenesis is modulated probably through the precise presence and maintenance of essential proteins in a time- and tissue-dependent manner [20, 23]. Empirical evidence shows that expression of ciliary genes is transcriptionally regulated and some transcriptional factors involved in ciliogenesis have been identified. These transcription factors include: HNF1 $\beta$ (hepatocyte nuclear factor 1 $\beta$ ) [24], FKH-2 (forkhead 2) [25], RFX family of transcription factors [26], and FOXJ1 transcription factors [27]. Among them, RFX and FOXJ1 are two major transcriptional factors that control ciliogenesis. RFX proteins function as transcriptional regulators that interact with the X-box motif at MHC class II gene promoters [26, 28]. Functional analyses of RFX regulators indicate that they are required for modulating expression of key genes involved in different stages of ciliogenesis, including formation of ciliated sensory neurons, basal body migration and membrane docking, intraflagellar transport and ciliary motility [29-32].

FOXJ1 belongs to the forkhead/winged-helix family of transcriptional factors [23]. Loss-of-function analyses of Foxj1 demonstrate the requirement of this gene for biosynthesis of motile cilia in mouse tissues [33, 34]. A number of target genes of FOXJ1 that are involved in ciliary motility have been identified in model organisms such as zebrafish and Xenopus. This suggests that FOXJ1 is a master transcriptional factor of motile ciliogenesis [35, 36]. 
Putative SOX5 binding site

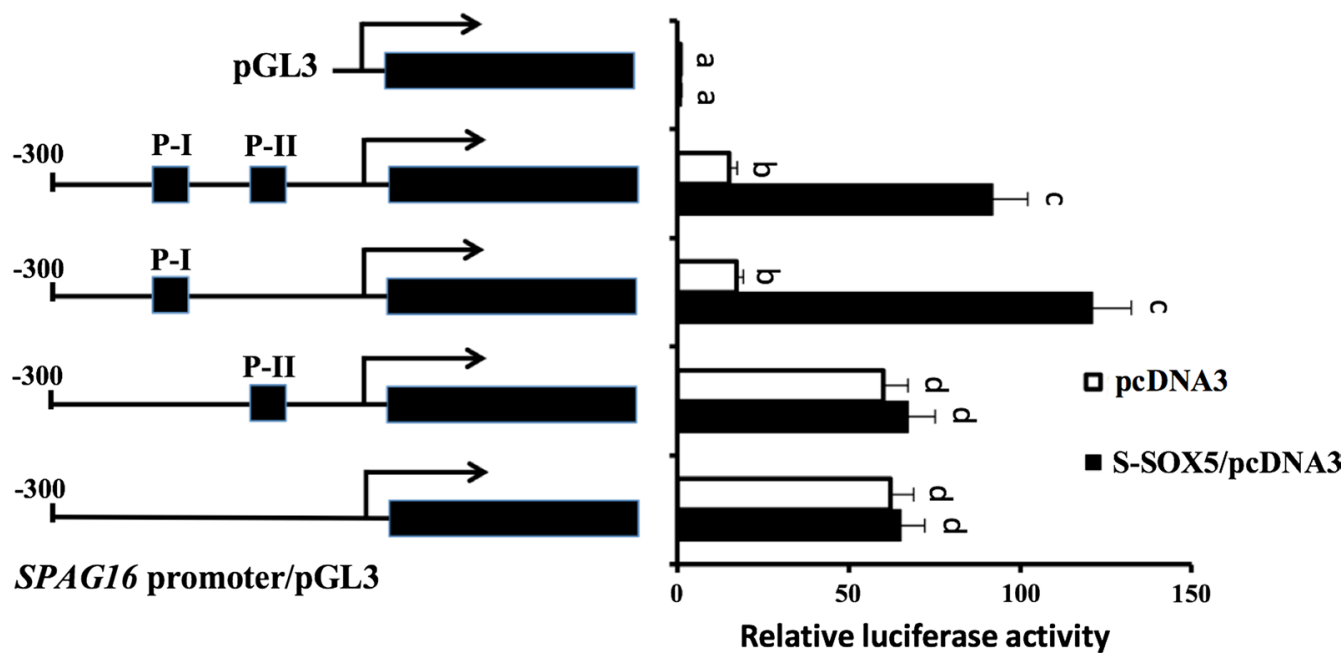

Fig. 5 Functional analyses of S-SOX5 binding sites in the human SPAG16L promoter. Left maps of the wild-type human SPAG16L promoter construct containing two putative SOX5 binding sites (P-I \& P-II) and the constructs with mutations of the potential SOX5 binding sites. Right, effect of S-SOX5 on the function of the wild-type and mutated SPAG16L promoter. BEAS-2B cells were co-transfected with SPAG16L promoter constructs and either pcDNA3 control or S-SOX5/pCDNA3. Relative luciferase activity, normalized to pGL3 control plasmids co-transfected with pcDNA3 vectors. Values are mean \pm S.E. (error bars) of the relative luciferase activity $(n=3)$. Bars labeled with different letters are significantly different $(p<0.05$; two-way ANOVA with Tukey's HSD test)

Our earlier studies demonstrated that S-SOX5, together with FOXJ1, regulates expression of an axonemal gene, Spag6 [9]. To explore if S-SOX5 also regulates other genes that are essential for motile cilia structure and/or function, and functions as a general transcription factor to control motile cilia/function, we decided to investigate if S-SOX5 regulates another axonemal central apparatus gene, SPAG16L, because the SPAG16L proximal promoter region also contains multiple putative SOX5 binding sites. Our findings demonstrated that S-SOX5 does regulate SPAG16L transcription through binding to the SOX5 binding sites. However, it should be aware that not all the putative SOX5 binding sites predicted by bioinformatic analysis are functional. One of the two putative SOX5 binding sites analysed in this study is not bound by S-SOX5. Thus, experiments must be conducted to verify if these putative binding sites are functional. Overall, this study presents another example that S-SOX5 regulates another gene essential for motile cilia function, and supports the notion that S-SOX5 is a general transcription factor to control formation and function of motile cilia.

Sperm flagella are special motile cilia. During spermiogenesis, germ cells undergo dramatic morphological changes as they develop into functional sperm. These changes include formation of flagella. Sperm flagella contain a " $9+2$ " axoneme. Besides this core axoneme structure, other affiliated structures, including the fibrous sheath and outer dense fibers, are also assembled into the sperm flagella [37]. S-SOX5 was originally cloned from mouse testis [10] and it is able to activate transcription of a group of testis-related gene such as $\mathrm{I} \kappa \mathrm{B} \beta$, ZNF230 and Catsper1 [12, 13, 38] Given that S-SOX5 is only expressed in tissues with motile cilia, particularly in the post-meiotic round spermatids [11], we hypothesize that this transcription factor regulates a suite of genes for motile cilia formation/function, particularly for sperm flagella formation/function. Recent GWAS studies suggest that the SOX5 locus is associated with male infertility [39], and the high expression level of S-SOX5 in the testis implies that S-SOX5 plays an important role in regulating expression of the genes that are essential for sperm function and male fertility.

The in vivo function of S-SOX5 is still not known. However, the unique exon not present in L-SOX5 allows us to make mutant mice with disruption of S-SOX5 only. Using this model, we will be able to study the function of S-SOX5 in vivo, and probably identify the target genes regulated by S-SOX5 globally.

\section{Conclusions}

This study demonstrates the molecular mechanism underlying the regulation of human SPAG16L by S-SOX5. S-SOX5 activates transcription of SPAG16L 
through specifically interacting with SOX5 binding sites at the SPAG16L promoter. The data suggest that S-SOX5 plays a regulatory role in the formation of cilia/flagella.

\section{Methods \\ Luciferase reporter constructs}

The SPAG16L promoter luciferase reporter fusion was constructed by cloning a $2 \mathrm{~kb}$ human $S P A G 16 L$ proximal promoter region including the transcriptional start site and multiple putative SOX5 binding sites into the pGL3basic vector (Cat.E1751, Promega). Similarly, another luciferase reporter fusion was made by cloning a shorter region of $S P A G 16 L$ proximal promoter into the pGL3basic vector. This transcriptional luc fusion construct contained the transcriptional start site of SPAG16L and adjacent two putative SOX 5 binding sites. The primers for construction of the transcriptional luc fusions were listed in Table 1.

\section{Expression constructs or adenovirus expressing S-SOX5 and SOX5 RNAi constructs}

S-SOX5 expression plasmids, the adenovirus expressing $S$-SOX5, and the RNAi constructs targeting SOX5 transcripts were generated previously in the laboratory [9]. Oligonucleotides used for generation of these constructs are listed in Table 1.
Site-directed mutagenesis of SOX 5 binding sites in the SPAG16L promoter

Two SOX5 binding sites in the SPAG16L promoter construct were mutated using a QuikChange II XL Site-Directed Mutagenesis Kit (Agilent Technologies) following the manufacturer's instructions. Mutations at the SPAG16L promoter were verified by DNA sequencing. The mutagenic primers for construction of mutated SOX5 binding sites are shown in Table 1.

\section{Western blot analysis}

Equal amount of proteins $(50 \mu \mathrm{g} / \mathrm{lane})$ were heated to $95^{\circ} \mathrm{C}$ in sample buffer for $10 \mathrm{~min}$, resolved in $10 \%$ SDSpolyacrylamide gels and then electro-transferred to polyvinylidene difluoride membranes (Millipore). After blocking in TBS-T buffer (Tris-buffered saline solution containing $5 \%$ non-fat dry milk and $0.05 \%$ Tween 20 ) for $1 \mathrm{~h}$, the membranes were incubated with antibodies against SOX5 (Aviva Systems Biology, Santa Cruz, CA) or rabbit $\beta$-actin (Cell Signaling Technology, Danvers, MA) overnight at $4{ }^{\circ} \mathrm{C}$. After being washed in TBS-T, the membranes were incubated with an anti-rabbit immunoglobulin conjugated with horseradish peroxidase (1:2000 dilution) at room temperature for $1 \mathrm{~h}$. SOX 5 or $\beta$-actin proteins were detected with SuperSignal West Pico Chemiluminescent Substrate (Pierce).

Table 1 Oligonucleotides used in this study

\begin{tabular}{|c|c|c|}
\hline Primer name & Sequence $\left(5^{\prime}-3^{\prime}\right)$ & Application \\
\hline SPAG16L-promoterF1 & GGTACCGGTCAAAGCGAAAGAAAACC & Forward primer for transcription fusion to luc \\
\hline SPAG16L-promoterR2 & CTCGAGGAACAGCGAAGACGCTACCC & Reverse primer for transcription fusion to luc \\
\hline Human S-SOX5 forward & GTGCCATAGGAGCTGTGCATG & S-SOX5 expression \\
\hline Human S-SOX5 reverse & GTTGGTCCTTCATTTGCCGAGC & \\
\hline SOX5 RNAi (225) sense & AAAAATGATGCTGTCACCAAGGCAA & SOX5 RNAi construction \\
\hline SOX5 RNAi (225) anti-sense & AAAGTTGCCTTGGTGACAGCATCAT & \\
\hline SOX5 RNAi (1109) sense & AAAAGATTATGGGAGTGACAGTGAA & SOX5 RNAi construction \\
\hline SOX5 RNAi (1109) anti-sense & AAAGTTCACTGTCACTCCCATAATC & \\
\hline SPAG16L RT-PCRF & TTCAGACTGCTGCTTCCATC & Real-time PCR analysis of SPAG16L \\
\hline SPAG16L RT-PCRR & TCGCCTGTACATAGATCCCA & \\
\hline GAPDH RT-PCRF & GGAGGTGAAGGTCGGAGTC & Real-time PCR analysis of GAPDH \\
\hline GAPDH RT-PCRR & GAAGATG GTGATGGGATTTC & \\
\hline SPAG16L mutation 1F & TGCAATGCAAGCCAACCACCTACTGTATCTTGTCC & Mutation of SOX5 binding site (P-I site) at SPAG16L promoter \\
\hline SPAG16L mutation 1R & GGACAAGATACAGTAGGTGGTTGGCTTGCATTGCA & \\
\hline SPAG16L mutation 2F & GTTAACTAGGCAACACTACCGCCACGGTAACTGGG & Mutation of SOX5 binding site (P-II site) at SPAG16L promoter \\
\hline SPAG16L mutation 2R & CCCAGTTACCGTGGCGGTAGTGTTGCCTAGTTAAC & \\
\hline SPAG16L ChIP-aF & CTTGGGTGACTTCCAATTTTG & ChIP assays for SPAG16L site a \\
\hline SPAG16L ChIP-aR & GTATCATTAACTACACTCCTC & \\
\hline SPAG16L ChIP-bF & CCTACTTGAGGAGGAGAGTGGGA & ChIP assays for SPAG16L site b \\
\hline SPAG16L ChIP-bR & TATCGCGTATGTATCAGAAGC & \\
\hline SPAG16L ChIP-CF & GGAAGATCCTCTCAGCAATAAGAC & ChIP assays for SPAG $16 \mathrm{~L}$ site c \\
\hline SPAG16L ChIP-cR & GAAGAACTATGGTGTTCAGC & \\
\hline
\end{tabular}




\section{Chromatin immunoprecipitation (ChIP)}

ChIP assays were conducted using a ChIP assay kit (Millipore) according to the manufacturer's instructions. Briefly, BEAS-2B cells were (CRL-9609) purchased from the American Type Culture Collection and infected with AdS-SOX 5 for $48 \mathrm{~h}$. After infection, the protein-DNA complexes from the cells were cross-linked by addition of $1 \%$ formaldehyde. The cells were suspended in SDS lysis buffer and were sonicated to shear DNA to 200-1000 bp fragments. Samples were precleared with protein A agarose/salmon sperm DNA ( $50 \%$ slurry) and were immunoprecipitated with antibodies against SOX5 or IgG. After washing the immunocomplexes with appropriate buffers, DNA was recovered by reverse cross-linking and purified by phenol/chloroform extraction followed by ethanol precipitation. The DNA fragments were used as a template for PCR reaction with primer sets (Table 1) flanking the SOX5 binding sites.

\section{Transient transfection and luciferase assays}

Human bronchial epithelial BEAS-2B cells were cultured in BEBM and were plated $24 \mathrm{~h}$ before transfection. The cells were transfected with plasmids containing the wild-type or mutated SPAG16 promoter using FuGENE6 transfection reagent (Roche). Co-transfection was performed with either empty vectors or S-SOX5 expression vectors (S-SOX5/pcDNA3). The cells were cultured for $48 \mathrm{~h}$ and the promoter activity was measured by the Dual-Luciferase Reporter Assay System (Promega). Luciferase activity was normalized to Renilla luciferase activity (control vector).

\section{Real-time PCR}

Total RNA was extracted from BEAS-2B cells infected with indicated plasmids using TRIzol Reagent (Invitrogen) and was reversed transcribed to cDNA. The cDNA was used for PCR amplification of SPAG16L, SOX5 and GAPDH with primers listed in Table 1. Real-time PCR was performed using $2 \times$ SYBR Green master mix (Bio-Rad).

\section{Abbreviations \\ SPAG16: sperm-associated antigen 16; ChIP: chromatin immunoprecipitation.}

\section{Authors' contributions}

$Z Z$ and $L Z$ designed the experiments, $L Z$ and $Y L$ wrote the manuscript. $L Z, W L$, $\mathrm{QZ}, Y L, J L, J M, Y L$ and CS performed the experiments and analyzed the data. SS. and ZZ interpreted the data and reviewed the paper. All authors read and approved the final manuscript.

\section{Author details}

${ }^{1}$ School of Public Health, Wuhan University of Science and Technology, Wuhan 430065, Hubei, China. ${ }^{2}$ Department of Obstetrics and Gynecology, Virginia Commonwealth University, Richmond, VA 23298, USA. ${ }^{3}$ Central China Normal University, Wuhan, Hubei 430000, China. ${ }^{4}$ Department of Computer Science, Wellesley College, Wellesley, MA 02481-5701, USA. ${ }^{5}$ Wuhan Hospital for the Prevention and Treatment of Occupational Diseases, Wuhan 430000, Hubei, China.

\section{Acknowledgements}

The authors thank Dr. Yu-Qin Shi and Dr. Ting Zhou for comments on the manuscript.

\section{Competing interests}

The authors declare that they have no competing interests.

\section{Availability of data and materials}

All data generated during this study are included in this article.

\section{Funding}

This research was supported by the National Institutes of Health $(\mathrm{NIH})$ [HD076257, HD090306], Virginia Commonwealth University Presidential Research Incentive Program (PRIP) and Massey Cancer Award (to Z.Z.), Natural Science Foundation of China [81571428 to Z.Z, 81300536 to L.Z.], Department of Hubei Province of China (WJ2015Q026 to L.Z.).

Received: 21 October 2016 Accepted: 24 January 2017

Published online: 31 January 2017

\section{References}

1. Kamachi Y, Kondoh H. Sox proteins: regulators of cell fate specification and differentiation. Development. 2013;140(20):4129-44

2. Sarkar A, Hochedlinger $K$. The sox family of transcription factors: versatile regulators of stem and progenitor cell fate. Cell Stem Cell. 2013:12(1):15-30.

3. Bowles J, Schepers G, Koopman P. Phylogeny of the SOX family of developmental transcription factors based on sequence and structural indicators. Dev Biol. 2000:227(2):239-55.

4. Harley VR, Lovell-Badge R, Goodfellow PN. Definition of a consensus DNA binding site for SRY. Nucleic Acids Res. 1994;22(8):1500-1.

5. Kondoh H, Kamachi Y. SOX-partner code for cell specification: regulatory target selection and underlying molecular mechanisms. Int J Biochem Cell Biol. 2010;42(3):391-9.

6. Wegner M. All purpose Sox: the many roles of Sox proteins in gene expression. Int J Biochem Cell Biol. 2010;42(3):381-90.

7. Wunderle VM, Critcher R, Ashworth A, Goodfellow PN. Cloning and characterization of SOX5, a new member of the human SOX gene family. Genomics. 1996:36(2):354-8.

8. Ikeda T, Zhang J, Chano T, Mabuchi A, Fukuda A, Kawaguchi H, Nakamura $\mathrm{K}$, Ikegawa S. Identification and characterization of the human long form of Sox5 (L-SOX5) gene. Gene. 2002;298(1):59-68.

9. Kiselak EA, Shen X, Song J, Gude DR, Wang J, Brody SL, Strauss JF 3rd, Zhang Z. Transcriptional regulation of an axonemal central apparatus gene, sperm-associated antigen 6, by a SRY-related high mobility group transcription factor, S-SOX5. J Biol Chem. 2010;285(40):30496-505.

10. Denny P, Swift S, Connor F, Ashworth A. An SRY-related gene expressed during spermatogenesis in the mouse encodes a sequence-specific DNA-binding protein. EMBO J. 1992;11(10):3705-12.

11. Connor F, Cary PD, Read CM, Preston NS, Driscoll PC, Denny P, CraneRobinson C, Ashworth A. DNA binding and bending properties of the post-meiotically expressed sry-related protein Sox-5. Nucleic Acids Res. 1994;22(16):3339-46.

12. Budde $L M$, Wu C, Tilman C, Douglas I, Ghosh S. Regulation of IKB $\beta$ expression in testis. Mol Biol Cell. 2002;13(12):4179-94.

13. Xu W, Zhang S, Qiu W, He G, Liu Y, Sun Y, Ma Y, Dong J, Zhang W. Spermatogenesis-related ring finger gene ZNF230 promoter: identification and functional analysis. Mol Biol Rep. 2009;36(5):1187-93.

14. Smith EF, Lefebvre PA. PF20 gene product contains WD repeats and localizes to the intermicrotubule bridges in Chlamydomonas flagella. Mol Biol Cell. 1997:8(3):455-67.

15. Pennarun G, Bridoux AM, Escudier E, Dastot-Le Moal F, Cacheux V Amselem S, Duriez B. Isolation and expression of the human hPF20 gene orthologous to Chlamydomonas PF20: evaluation as a candidate for axonemal defects of respiratory cilia and sperm flagella. Am J Respir Cell Mol Biol. 2002;26(3):362-70.

16. Zhang Z, Sapiro R, Kapfhamer D, Bucan M, Bray J, Chennathukuzhi V, et al. A sperm-associated WD repeat protein orthologous to Chlamydomonas 
PF20 associates with Spag6, the mammalian orthologue of Chlamydomonas PF16. Mol Cell Biol. 2002;22(22):7993-8004.

17. Nagarkatti-Gude DR, Jaimez R, Henderson SC, Teves ME, Zhang Z, Strauss JF 3rd. Spag 16, an axonemal central apparatus gene, encodes a male germ cell nuclear speckle protein that regulates SPAG16 mRNA expression. PLoS ONE. 2011;6(5):e20625.

18. Zhang Z, Kostetskii I, Moss SB, Jones BH, Ho C, Wang H, et al. Haploinsufficiency for the murine orthologue of Chlamydomonas PF20 disrupts spermatogenesis. Proc Natl Acad Sci USA. 2004;101 (35):12946-51.

19. Zhang Z, Kostetskii I, Tang W, Haig-Ladewig L, Sapiro R, Wei Z, et al. Deficiency of SPAG 16L causes male infertility associated with impaired sperm motility. Biol Reprod. 2006;74(4):751-9.

20. Satir P, Christensen ST. Overview of structure and function of mammalian cilia. Ann Rev Physiol. 2007;69:377-400.

21. Badano JL, Mitsuma N, Beales PL, Katsanis N. The ciliopathies: an emerging class of human genetic disorders. Ann Rev Genom Human Genet. 2006;7:125-48.

22. Roy S. The motile cilium in development and disease: emerging new insights. BioEssays. 2009;31(7):694-9.

23. Thomas J, Morle L, Soulavie F, Laurencon A, Sagnol S, Durand B. Transcriptional control of genes involved in ciliogenesis: a first step in making cilia. Biol Cell/Auspices Eur Cell Biol Org. 2010;102(9):499-513.

24. Roelandt P, Antoniou A, Libbrecht L, Van Steenbergen W, Laleman W, Verslype C, et al. HNF1B deficiency causes ciliary defects in human cholangiocytes. Hepatology. 2012;56(3):1178-81.

25. Mukhopadhyay S, Lu Y, Qin H, Lanjuin A, Shaham S, Sengupta P. Distinct IFT mechanisms contribute to the generation of ciliary structural diversity in C. elegans. EMBO J. 2007;26(12):2966-80.

26. Reith W, Herrero-Sanchez C, Kobr M, Silacci P, Berte C, Barras E, Fey S, Mach B. MHC class II regulatory factor RFX has a novel DNA-binding domain and a functionally independent dimerization domain. Genes Dev. 1990;4(9):1528-40.

27. Mazet F, Yu JK, Liberles DA, Holland LZ, Shimeld SM. Phylogenetic relationships of the Fox (Forkhead) gene family in the Bilateria. Gene. 2003;316:79-89.

28. Reith W, Satola S, Sanchez CH, Amaldi I, Lisowska-Grospierre B, Griscelli C, Hadam MR, Mach B. Congenital immunodeficiency with a regulatory defect in MHC class II gene expression lacks a specific HLA-DR promote binding protein, RF-X. Cell. 1988;53(6):897-906.
29. Dubruille R, Laurencon A, Vandaele C, Shishido E, Coulon-Bublex M, Swoboda P, Couble P, Kernan M, Durand B. Drosophila regulatory factor $X$ is necessary for ciliated sensory neuron differentiation. Development. 2002;129(23):5487-98.

30. Piasecki BP, Burghoorn J, Swoboda P. Regulatory Factor X (RFX)-mediated transcriptional rewiring of ciliary genes in animals. Proc Natl Acad Sci USA. 2010;107(29):12969-74.

31. Swoboda P, Adler HT, Thomas JH. The RFX-type transcription factor DAF-19 regulates sensory neuron cilium formation in C. elegans. Mol Cell. 2000;5(3):411-21.

32. Wu Y, Hu X, Li Z, Wang M, Li S, Wang X, et al. Transcription Factor RFX2 Is a Key Regulator of Mouse Spermiogenesis. Sci Rep. 2016;6:20435.

33. Brody SL, Yan XH, Wuerffel MK, Song SK, Shapiro SD. Ciliogenesis and leftright axis defects in forkhead factor HFH-4-null mice. Am J Respir Cell Mol Biol. 2000;23(1):45-51.

34. Jacquet BV, Salinas-Mondragon R, Liang H, Therit B, Buie JD, Dykstra M, et al. FoxJ1-dependent gene expression is required for differentiation of radial glia into ependymal cells and a subset of astrocytes in the postnatal brain. Development. 2009;136(23):4021-31.

35. Stubbs JL, Oishi I, Izpisua Belmonte JC, Kintner C. The forkhead protein Foxj1 specifies node-like cilia in Xenopus and zebrafish embryos. Nat Genet. 2008;40(12):1454-60.

36. Yu X, Ng CP, Habacher H, Roy S. Foxj1 transcription factors are master regulators of the motile ciliogenic program. Nat Genet. 2008;40(12):1445-53.

37. Teves ME, Nagarkatti-Gude DR, Zhang Z, Strauss JF 3rd. Mammalian axoneme central pair complex proteins: broader roles revealed by gene knockout phenotypes. Cytoskeleton. 2016;73(1):3-22.

38. Mata-Rocha M, Hernandez-Sanchez J, Guarneros G, de la Chesnaye E, Sanchez-Tusie AA, Trevino CL, Felix R, Oviedo N. The transcription factors Sox 5 and Sox 9 regulate Catsper1 gene expression. FEBS Lett. 2014;588(18):3352-60.

39. Hu Z, Xia Y, Guo X, Dai J, Li H, Hu H, et al. A genome-wide association study in Chinese men identifies three risk loci for non-obstructive azoospermia. Nat Genet. 2012;44(2):183-6.

\section{Submit your next manuscript to BioMed Central and we will help you at every step:}

- We accept pre-submission inquiries

- Our selector tool helps you to find the most relevant journal

- We provide round the clock customer support

- Convenient online submission

- Thorough peer review

- Inclusion in PubMed and all major indexing services

- Maximum visibility for your research

Submit your manuscript at www.biomedcentral.com/submit
() Biomed Central 